\title{
„Digitales Lernen und Lehren: Das neue Normal?““
}

\author{
Susanne Robra-Bissantz $\cdot$ Linda Grogorick
}

Angenommen: 20. Oktober 2021 / Online publiziert: 2. November 2021

(C) Der/die Autor(en) 2021

Was haben wir uns mit dieser Frage, formuliert mitten in der Pandemie, gedacht? Nehmen wir die Antworten, die uns unsere Beiträge aus dieser Ausgabe gegeben haben, direkt vorweg: Ein neues Normal sind digitales Lernen und Lehren nicht geworden.

Trotzdem. Es hat sich einiges getan und einige Hürden, die Bildungseinrichtungen vor der Pandemie nicht genommen haben, sind überwunden oder zumindest erkannt und angegangen.

Wir standen nicht gut da, in digitaler Bildung. Warum?

Vielleicht, weil wir sie nicht wirklich wollten. Als Lehrende oder auch Mitgestalter von Präsenzinstitutionen ist es schwer sich vorzustellen, dass plötzlich unsere Lernenden nicht mehr vor Ort sein sollen. Ist es nicht, in Schulen, gut gelebte Tradition und vielleicht, bei Hochschulen, ein Wettbewerbsfaktor, dass wir persönliche und reale Interaktionen untereinander pflegen? Ist, daneben, Schule, Hochschule, Aus- und Weiterbildung nicht mehr, als fachliches Wissen weiterzugeben - ist es nicht auch Gemeinschaft, gegenseitige Anregung und informelle Erfahrung?

Vielleicht konnten wir digitale Bildung in unseren Organisationen auch nicht richtig gut. Was soll vorteilhaft daran sein, für viel Geld Technik anzuschaffen, wenn man im Gegenzug die Vorteile, seien sie finanzieller Art oder in Bezug auf die Qualität der Bildung nicht (direkt) sieht?

Wir werden, in diesem Herausgeberband, sehen dass es richtig war, im Sinne der HMD die Wirtschaftsinformatikforschung mit Blick auf die Praxis zu befragen.

\footnotetext{
Susanne Robra-Bissantz $(\bowtie) \cdot$ Linda Grogorick

Lehrstuhl Informationsmanagement, Institut für Wirtschaftsinformatik, Technische Universität

Braunschweig, Mühlenpfordtstr. 23, 38106 Braunschweig, Deutschland

E-Mail: s.robra-bissantz@tu-bs.de

Linda Grogorick

E-Mail: 1.grogorick@tu-bs.de
} 
Denn es ist die Wirtschaftsinformatik, die den Unterschied zwischen zwei englischen Übersetzungen der Digitalisierung kennt. Es geht eben nicht um Digitization - die Überführung analoger Prozesse mithilfe von Technik in das Digitale. Sondern es geht um Digitalization: die Veränderung von Prozessen, Geschäfts- oder Bildungsmodellen und Organisationen so, dass die Potenziale der digitalen Technik geborgen werden. Und es ist auch die Wirtschaftsinformatik, die mit dem Blick auf das gesamte Informationssystem aus Aufgabe (Lernen und Lehren), Technik und Mensch (auch in der Organisation) weiß, was alles in einer Digitalization zu berücksichtigen ist.

Wir danken unseren Autor:innen für Beiträge, die

- Erfolgsfaktoren oder Gestaltungstipps für die digitale Lehre aus dem Blickwinkel der Student:innen, einzelner Medienformen oder der Organisation erarbeiten,

- Vorteile des digitalen Lernens in verschiedenen Phasen des Lernprozesses erkennen, analysieren und entsprechend ausprägen,

- Lösungsvorschläge für eine hybride Lehre in flexibel einsetzbaren virtuellen und realen Räumen aufzeigen und

- mit Blick über die einzelne Institution hinaus die Vorteile gemeinsamer Lehrkonzepte erarbeiten und diese auch entwickeln.

Denn diese Beiträge beschreiben Wege zu einem neuen Normal - wie auch immer dieses aussehen wird -

- über stetige Verbesserung der digitalen Lehre,

- über zielgerichteten Einsatz digitaler Lehre sobald sie das Lernen, z. B. über Individualisierung oder Kollaboration, verbessert,

- über kanalunabhängige Konzepte, die sowohl in Präsenz als auch digital funktionieren und damit dem Lehrenden ermöglichen, auf die aktuelle Situation (z.B. räumliche Verfügbarkeit und Bedürfnisse der Lernenden) zu reagieren oder in Lernphasen zwischen dem physischen und digitalen Raum zu wechseln,

- oder über neue, durch Digitalisierung ermöglichte Ansätze der Zusammenarbeit zwischen Lehrenden, von wiederverwendbaren Gestaltungsansätzen bis zu zentralen Plattformen für Bildungsinhalte.

Wir sind gespannt, was Bildungsinstitutionen und damit auch wir in Zukunft als „normales“ Lernen und Lehren empfinden.

Doch nun zum Heft, im Einzelnen. Wir beginnen mit einem Grundlagenbeitrag. Dieser zeigt zunächst theoretisch auf, wie digitale Medien heute über jeweils veränderte Aufgaben und raffinierte Lernformen in der Lage sind, verschiedene, auch moderne Lerntheorien zu stützen. Aus praktischer Sicht stellt er mithilfe aktueller Studien die derzeitige Situation in Hochschulen, Schulen sowie in der (betrieblichen) Aus- und Weiterbildung dar.

Unser erster Schwerpunktbeitrag von Gergana Vladova et al. geht genauer auf die Situation an Hochschulen ein: fokussiert auf die Studierendenperspektive, auf der Grundlage des Lehr-/Lern-Transaktionsmodells. Aus einer Studie mit über 800 Teilnehmer:innen leitet er Gestaltungsempfehlungen und konkrete Tipps für z.B. Interaktion der Lehrenden mit den Lernenden, für Lernende selber sowie für weitere 
Aspekte, die zum Erfolg des Lernens beitragen (z.B. Lernstil und Lernumgebung) $\mathrm{ab}$.

Im weiteren geht Elske Ammenwerth auf besondere Erfolgsfaktoren digitaler Lehre ein. Der Beitrag zeigt wiederum mit Tipps und einfach umzusetzenden Gestaltungsempfehlungen auf, wie die Aktivierung von Studierenden sowie Interaktionen zwischen allen Beteiligten auch virtuell gelingen. Dabei weist die Autorin darauf hin, dass in der Digitalisierung der Lehre das Potenzial liegt, eine neue auf den Lernenden fokussierte Lernphilosophie ,from Teaching to Learning“ umzusetzen.

Tobias Teich et al. bezeichnen diesen neuen, autonomen und individuellen Wissenserwerb als New Learning, das ähnlich wie heute New Work die Unternehmen prägen wird. Auch sie betonen die im konnektivistischen Sinn wesentlichen Interaktionen, die gemeinschaftliches Lernen ermöglichen. Entsprechend stellen sie, am Beispiel einer IT-Schulung, digitale Gestaltungsansätze für Interaktionen zwischen Teilnehmenden, zwischen Lerner:in und Betreuer:in sowie zwischen Betreuenden vor.

Fokussiert man nun - auf dem Weg vom Lehren zum Lernen - die Möglichkeit des Lernenden sich individuell durch Inhalte zu bewegen, so werden die individuellen Interaktionen zur Begleitung dieses Lernprozesses schnell aufwändig. Michael Striewe et al. zeigen daher für das Thema der grafischen, konzeptuellen Modellierung auf, wie in der individuellen Betreuung die Überprüfung von Übungsaufgaben automatisiert gelingt. Gelungen fundiert, beispielsweise über eine detaillierte Analyse von zu erwerbenden Kompetenzen und Anforderungen an die technische Unterstützung, gelangt die Forschungsgruppe aus vielen Partnern zu einer übertragbaren Lösung.

Die nächsten Forschungsgruppen widmen sich insbesondere den Chancen, die durch die ganz besonderen Interaktionen in einer Kooperation und Kollaboration zwischen den Lernenden entstehen. Auch digital. Obwohl uns eine digitale Zusammenarbeit vor der Pandemie kaum möglich vorkam.

Als erste Erkenntnis hierzu tragen Pia Gebbing et al. bei, dass es durchaus gelingt, mithilfe von besonders ausgestalteten Whiteboards zu kooperieren und dabei die zukünftig wesentliche Kompetenz der Kreativität zu fördern. Allerdings ist es hierzu notwendig tief in Bedürfnisse und Präferenzen der beteiligten Menschen einzusteigen und mithilfe der Technologie neben den notwendigen Features zur kreativen Arbeit (wie z. B. Synchronizität oder Reichhaltigkeit) auch ganz individuelle Werte der digitalen Zusammenarbeit zu berücksichtigen. So kann ein digitales Whiteboard beispielsweise die oft gewünschte Anonymität und Rückzugsmöglichkeit ebenso wie gegenseitige Sichtbarkeit ermöglichen, geht aber häufig auch mit Techno-Stress einher.

Eingebettet in ein sowohl aus technischer als auch didaktischer Sicht wohl geplantes, weiterhin stetig evaluiertes und übertragbares digitales Veranstaltungskonzept aus der Wirtschaftsinformatik zeigen Jens Kaufmann et al. auf, wie kollaborative Live-Sessions zur Übung von Inhalten an einem virtuell geteilten Bildschirm von Studierenden sehr gut angenommen werden und zu ihrer Motivation beitragen. Als hybride Lösung sehen sie die Vorteile digitaler Kollaboration auch in zukünftigen Präsenz-Settings. Dabei weisen sie zusätzlich darauf hin, dass digitale Kollaboration nicht nur möglich, sondern ein Kompetenzerwerb in diesem Bereich auch dringend 
für zukünftige Arbeitswelten nötig ist - eine Win-Win-Situation für die digitale kollaborative Lehre.

Gerade letzterer Aspekt findet sich auch im folgenden Beitrag von Julian Hofer und Ralf Knackstedt, die sich mit problemlösungsorientiertem Lernen als Vorbereitung auf die in der Wirtschaftsinformatik-Praxis typische Projektarbeit beschäftigen. Die Kompetenz zur digitalen Kollaboration wird hier zukünftig potenziell immer wichtiger und damit zum Lernziel. Hofer und Knackstedt zeigen auf, dass das heutige agile Vorgehen auch digital sehr gut funktioniert und geben aus ihrer Erfahrung mit virtuellen IT-Studienprojekten konkrete Handlungsempfehlungen, die sich beispielsweise darauf beziehen, den Studierenden durch geeignete Unterstützungssystem eigenständige Entscheidungen sowie persönliche Nähe zu ermöglichen.

Auch Florian König et al. beschäftigen sich mit dem kollaborativen, problemlösungsorientierten Lernen für die typische IT-Projektarbeit. Ihr Format des Hackathon wurde bereits in der Präsenzlehre mit realer Gruppenarbeit an einem Wochenende durchgeführt. Dank der sorgfältigen Planung eines etwas kürzeren, digitalen Hackathon-Wochenendes stellte sich in der Pandemie heraus, dass das Format des Hackathon gut in die digitale Lehre übertragbar ist und die Evaluation für beide Formen (Präsenz und virtuell) ähnliche, weitere Gestaltungsempfehlungen ergibt. Damit ist der Hackathon ebenso wie die im vorherigen Artikel beschriebene agile Projektarbeit ein kanalunabhängiges Format, das (gemäß aber auch aufgrund der Erkenntnisse aus den Beiträgen) im digitalen Raum ebenso wie in Präsenz stattfinden kann. Als interessante Erkenntnis ergibt sich, dass Lehrende für die Zukunft in ihre Entscheidung für ein Präsenz- oder digitales Format einbeziehen sollten, ob sie der informellen sozialen Interaktion des realen Raums oder dem Kompetenzerwerb in digitaler Kollaboration eine höhere Wichtigkeit zuweisen.

Jenseits der Vorbereitung auf eine IT-Projektarbeit zeigen Alina Bockshecker et al. am Beispiel eines Kurses zum komplexen und vielfältig diskutierten Thema der digitalen Transformation auf, dass problembasiertes Lernen in Gruppen zum einen das Lernen und insbesondere die Lernaktivität von Studierenden verbessert und zum anderen, wie in den vorhergehenden Beiträgen, kanalunabhängig sowohl mit Präsenzphasen als auch rein digital umsetzbar ist. Dazu stellen die Autor:innen ihr evaluiertes und übertragbares Lernkonzept anhand eines Prozessmodells des problembasierten Lernens, angereichert durch zusätzlich motivierende Spielelemente (wie beispielsweise eine Geschichte, Rollen oder Abzeichen), vor. Als Erkenntnis aus der mehrfachen digitalen Durchführung weisen sie auf die Wichtigkeit der Betreuung der Gruppenarbeit durch Lehrende hin.

Für alle vorgestellten, kanalunabhängigen Konzepte stellt sich die Frage, wie Vorteile des digitalen Lernens, wie z.B. die zeitliche Autonomie und die häufig besser umsetzbare Kollaboration, zwischen Studierenden mit den Vorteilen der Präsenzlehre mit z.B. potenziell mehr sozialen Interaktionen je nach Situation (z.B. in der Pandemie) verknüpft werden können. Detlef Stern stellt hierzu sein Konzept des Agilen Studierens vor, das die Konzepte der agilen Software-Entwicklung auf die Lehre überträgt und damit eine neue Sicht auf den Lernenden und die Organisation des Lernens bietet. Statt den Lernenden mit vorbereiteten Inhalten in einem Lehrplan zu konfrontierten, stellt der Lehrende die Lernenden in das Zentrum des Interesses und reagiert auf ihren gewünschten Wissenserwerb und ihre veränderlichen Lernpro- 
bleme. Verschiedene Phasen der reinen Wissensvermittlung, der Themenwahl durch Studierende, der Bearbeitung dieser Themen in Studierendengruppen und der Diskussion mit Lehrenden können sowohl in Präsenz als auch digital erfolgen. Dabei zeigt der Beitrag auf, wie die hybride Mischung vor der Pandemie sowie in ihren verschiedenen Phasen jeweils relativ einfach umstell- und umsetzbar war.

Auch die Weiterbildung in Unternehmen mit ihren zunehmenden und vielfältigen Bildungsbedarfen muss digitaler werden und sich gleichzeitig hin zu einer konsequenten Berücksichtigung der individuellen Bildungsbedarfe der Mitarbeiter:innen wandeln - verbunden mit einer Veränderung der organisatorischen Rahmenbedingungen. Oliver Gast et al. schlagen hierzu ein Human Experience Design des Lernens vor, das Ansatzpunkte zur mensch-zentrierten digitalen Transformation in der Organisation, wie beispielsweise die Analyse und das Umdenken in Bezug auf das Führungsverhalten, mit einem Blick auf den Mitarbeitenden und z. B. seine Motivation und Fähigkeit zur Nutzung digitaler Bildungsangebote verknüpft.

Aber Digitalisierung stellt nicht nur Anforderungen an die Organisation des Lernens sondern sie eröffnet auch Chancen, wenn digitale Module hochschulübergreifend gestaltet und angeboten werden können. Ralf Plattfaut et al. stellen hierzu ein positiv von Studierenden evaluiertes Lehr-/Lernkonzept zum Geschäftsprozessmanagement vor, das an zwei Hochschulen gleichzeitig angeboten wurde und damit eine Kooperation zwischen den Lehrenden in der Entscheidung über sowie der Erstellung von Kursmaterialien bis hin zu einer gemeinsam erstellten Prüfung ermöglichte. Neben Klärung der technologischen Voraussetzungen halten die Autor:innen vor allem das gegenseitige Vertrauen der beteiligten Lehrenden für wichtig. Sie verweisen darauf, dass das Lernangebot in Zukunft sowohl für weitere Hochschulen verfügbar gemacht werden als auch Eingang in eine zentrale Bildungsplattform, z. B. des Landes, finden kann.

Genau diesen Weg von digitalen Modulen für eine oder mehrere Organisationen hin zu einer Service-Plattform für Bildungsangebote hat das im nächsten Beitrag von Jannick Eckle et al. beschriebene Projekt School to go für Schulen beschritten. Es ergreift die Chancen der Digitalisierung durch eine Social-Media-orientierte, partizipative Plattform, die Lernenden und Lehrenden gleichermaßen Vorteile bietet. Lehrende greifen auf die einzelnen Module zu, sie erhalten Tipps für die Durchführung und tauschen sich auf der Plattform aus. Lernende können ebenso eigenständig ihre Lernangebote wählen und erhalten zudem, wie in anderen digitalen ServiceÖkosystemen, individuelle Empfehlungen.

Der letzte Schwerpunktbeitrag dieser HMD führt viele Wege zu einem neuen Normal in der digitalen Bildung zusammen. Tim Weinert et al. zeigen auf, wie sie die digitale Lehre am Beispiel einer Lernform - dem Lernvideo - verbessern, indem sie dieses mit interaktiven und gamifizierten Elementen anreichern. Dabei fokussieren sie insbesondere, wie die digitalen Elemente zu einer höheren Motivation und einem erhöhten Lernerfolg führen können und tragen hierzu sowohl bestehende theoretische als auch praktische Erkenntnisse aus Studierenden- und Anbieterperspektive zusammen. Ziel der gestaltungsorientierten Forschung sind dann Entwurfsmuster, mit denen beliebige Lehrende oder andere Stakeholder durch die Gestaltung interaktiver gamifizierter Lernvideos geleitet werden. Damit wird die besondere Chance der Digitalisierung ergriffen, nicht allein Ergebnisse eines Gestaltungsprozesses (hier 
z. B. eines digitalen Kurses), sondern grundlegendes Wissen zum Design dieser Ergebnisse für viele potenziell Interessierte verfügbar zu machen.

Abschließend verbleibt uns zu unseren Schwerpunktartikeln zu vermerken, dass uns für dieses Heft viele Beiträge aus Hochschulen erreicht haben, die von ihrer Wirtschaftsinformatik-Forschung für ihre eigene Lehr-Praxis berichten. Die Rezension eines Sammelbands, der verschiedene Perspektiven auf die tatsächliche, veränderte Situation durch Corona an Hochschulen der Länder Deutschland, Österreich und Schweiz bietet, rundet diesen Schwerpunkt im Schwerpunkt ab. Wir hoffen und glauben trotzdem, dass die vorliegenden Einblicke in die Entwicklungen des Lernens und Lehrens während und nach einer Zeit, die von vielen ganz plötzlich forderte, mit der Digitalisierung zurecht zu kommen, ihre Chancen und Potenziale zu nutzen oder sogar in eine mehr oder weniger digitale Zukunft zu tragen, auch für Leser:innen aus der Praxis der Schule oder der Weiterbildung wichtige Gestaltungshinweise, Tipps oder Anregungen sein können.

Zudem machen wir Sie gerne aufmerksam auf insgesamt vier Beiträge in der Rubrik Spektrum, die sich mit völlig anderen Themen als der digitalen Bildung beschäftigen.

Zwei davon behandeln das Verhalten von privaten Kunden in Bezug auf den Datenschutz.

Julian Kettl et al. tragen hier mit einer empirischen Studie in Form einer ConjointAnalyse bei, dass sich Studierende bei ihrer Entscheidung für ein Online-Girokonto von wenigen Eigenschaften des Angebots leiten lassen: vor allem von der Grundgebühr sowie von Datenschutz \& Privatheit. Dabei wird zusätzlich letzterem Kriterium ein deutlich höherer Wert zugewiesen, sodass Studierende bereit sind deutlich mehr für einen sichereren Dienst zu bezahlen. Unterschiede in dieser Beurteilung hängen von verschiedenen Faktoren wie der Vorerfahrung mit Online-Finanzdienstleistungen oder dem Geschlecht der Person ab.

Aus theoretischer Sicht konstatieren Wassili Lasarov und Stefan Hoffmann auf Basis einer umfassenden Literaturstudie ein paradoxes Datenschutzverhalten von Konsument:innen. Während sie in ihrer Einstellung rational zwischen den Kosten (der Freigabe von Daten) und dem Nuten (z.B. der Personalisierung) unterscheiden, verhalten sie sich doch häufig aufgrund von situativen Einflüssen (z. B. wenig Zeit) oder kognitiven Verzerrungen (z.B. einer Kontrollillusion) wenig vorsichtig. Die Autoren strukturieren die Diskrepanz zwischen Einstellung und Verhalten in Gaps zwischen erstrebenswerter, erreichbarer und erstrebter Privatsphäre, schlagen vor, diese genauer zu analysieren und geben jeweils bereits interessante, praxisnahe Beispiele.

Der Beitrag von Leon Adler et al. fokussiert wiederum den Schutz von Daten diesmal aus Sicht kooperierender Unternehmen, die im Rahmen von Industrie 4.0 Daten beispielsweise zur Nutzung von Produkten oder zu Materialdetails austauschen. Die Autor:innen entwickeln ein Vorgehensmodell dazu, wie Unternehmen diejenigen Daten identifizieren können, die für das eigene Geschäft kritisch sind und daher besser nicht weitergegeben werden sollten und wenden dieses anschaulich an einem Beispiel an.

Sebastian Floerecke beschäftigt sich mit IT-Service-Katalogen und dem Angebot von IT-Services in Self-Service-Portalen. Im Rahmen einer Fallstudie gelangt 
er mithilfe bestehender Literatur sowie Erfahrungsberichten zu Ansätzen dafür, wie das Angebot von IT-Services für interne oder externe Mitarbeiter:innen kundengerechter gestaltet werden kann. In Bezug auf Self-Service-Portale stellt er - für Unternehmensentscheider und IT-Verantwortliche - Kriterien und Anforderungen zusammen, die diesen bei Auswahl, Integration und Customizing helfen.

Unser Heft schließt letztlich mit einem Beitrag aus unserer neuen Rubrik: Forschung für die Praxis. In dieser Rubrik zeigen Autoren auf, wie es mithilfe geschickter Forschungsmethoden gelingt, Forschungsergebnisse zu erzielen, die Partner aus der Praxis direkt in ihren Unternehmen oder Organisationen umsetzen können.

Der Beitrag in diesem Heft beschäftigt sich mit der individuellen Digitalisierungsbereitschaft. Der Autor arbeitet mit Experteninterviews, die auf einer vorhergehenden Literaturanalyse basieren und gezielt individuelle Faktoren der Digitalisierungsbereitschaft sowie Möglichkeiten, diese zu fördern, thematisieren. Damit gelangt Manuel Muehlburger zum einen zu allgemeinen Ansätzen, wie beispielsweise Ängste vor Technologien abzubauen oder den Nutzen dieser aufzuzeigen, zum anderen aber auch zu Tipps für Initiativen, wie beispielsweise Schulungskonzepten, um die individuelle Digitalisierungsbereitschaft zu erhöhen. Zwar ist das digitale Lernen und Lehren nicht direkt angesprochen, die Erkenntnisse sind jedoch sicherlich ausgesprochen wertvoll für Bildungsinstitutionen.

Wir wünschen damit allen Leser:innen aus Bildungsinstitutionen ebenso wie aus Unternehmen, ob mit oder ohne Weiterbildung, viel Freude und viel Erkenntnis beim Stöbern, Lesen und Entdecken.

Susanne Robra-Bissantz und Linda Grogorick

Funding Open Access funding enabled and organized by Projekt DEAL.

Open Access Dieser Artikel wird unter der Creative Commons Namensnennung 4.0 International Lizenz veröffentlicht, welche die Nutzung, Vervielfältigung, Bearbeitung, Verbreitung und Wiedergabe in jeglichem Medium und Format erlaubt, sofern Sie den/die ursprünglichen Autor(en) und die Quelle ordnungsgemäß nennen, einen Link zur Creative Commons Lizenz beifügen und angeben, ob Änderungen vorgenommen wurden.

Die in diesem Artikel enthaltenen Bilder und sonstiges Drittmaterial unterliegen ebenfalls der genannten Creative Commons Lizenz, sofern sich aus der Abbildungslegende nichts anderes ergibt. Sofern das betreffende Material nicht unter der genannten Creative Commons Lizenz steht und die betreffende Handlung nicht nach gesetzlichen Vorschriften erlaubt ist, ist für die oben aufgeführten Weiterverwendungen des Materials die Einwilligung des jeweiligen Rechteinhabers einzuholen.

Weitere Details zur Lizenz entnehmen Sie bitte der Lizenzinformation auf http://creativecommons.org/ licenses/by/4.0/deed.de. 